\title{
Unstable Slip Pulses and Earthquake Nucleation as a Nonequilibrium First-Order Phase Transition
}

\author{
Efim A. Brener, ${ }^{1, *}$ Michael Aldam, ${ }^{2, *}$ Fabian Barras, ${ }^{3}$ Jean-François Molinari, ${ }^{3}$ and Eran Bouchbinder ${ }^{2}$ \\ ${ }^{1}$ Peter Grünberg Institut, Forschungszentrum Jülich, D-52425 Jülich, Germany \\ ${ }^{2}$ Chemical and Biological Physics Department, Weizmann Institute of Science, Rehovot 7610001, Israel \\ ${ }^{3}$ Civil Engineering Institute, Materials Science and Engineering Institute, Ecole Polytechnique Fédérale de Lausanne, \\ Station 18, CH-1015 Lausanne, Switzerland
}

(Received 18 July 2018; revised manuscript received 20 September 2018; published 7 December 2018)

The onset of rapid slip along initially quiescent frictional interfaces, the process of "earthquake nucleation," and dissipative spatiotemporal slippage dynamics play important roles in a broad range of physical systems. Here we first show that interfaces described by generic friction laws feature stressdependent steady-state slip pulse solutions, which are unstable in the quasi-1D approximation of thin elastic bodies. We propose that such unstable slip pulses of linear size $L^{*}$ and characteristic amplitude are "critical nuclei" for rapid slip in a nonequilibrium analogy to equilibrium first-order phase transitions and quantitatively support this idea by dynamical calculations. We then perform $2 \mathrm{D}$ numerical calculations that indicate that the nucleation length $L^{*}$ exists also in 2D and that the existence of a fracture mechanics Griffith-like length $L_{G}<L^{*}$ gives rise to a richer phase diagram that features also sustained slip pulses.

DOI: 10.1103/PhysRevLett.121.234302

Introduction.-The spatiotemporal dynamics of frictional interfaces ("faults"), formed when two deformable bodies come into contact, are central to a broad range of physical systems [1-3]. Two basic recurring themes, which still resist a complete theoretical understanding, are rapid slip nucleation and the rupture modes of faults. The former addresses the conditions under which slowly driven or strictly quiescent faults spontaneously develop rapid slip, the so-called "earthquake nucleation" problem [4-21]. The latter addresses the ways in which such faults rupture once rapid slip nucleates, in particular, the existence and properties of expanding cracklike rupture vs spatially compact pulse modes [21-33].

Earthquake nucleation has been extensively studied [4-21]. It has been shown that for a broad class of interfaces where the frictional resistance decreases with increasing slip velocity, i.e., in the velocity-weakening regime, nucleation emerges from a frictional instability [4-21]. This nucleation scenario, controlled by a critical nucleation length $L_{c}$ [34], assumes that external driving forces bring the interface or part of it into the destabilizing velocity-weakening regime, which is valid only above some typically low slip velocity. Far less is known about nucleation from the quiescent, nearly locked state that is generically velocity strengthening [46].

Once rapid slip commences, the spatiotemporal dynamics of frictional interfaces are largely determined by the mode of rupture propagation along them, e.g., [2,7]. While expanding cracklike rupture has been thought to be the dominant mode of rupture, it has been suggested that some earthquake data might be explained in terms of slip pulses [47]. This suggestion has triggered various $2 \mathrm{D}$ analyses [22-26] that demonstrated the existence of slip pulses for a class of friction models that feature aging or healing in the absence of slip and sufficiently strong velocity-weakening behavior. Recently, the existence of steady-state slip pulses in a class of generalized friction models has been demonstrated in the framework of the quasi-1D approximation of thin elastic bodies in contact [33]. Yet, the degree of generality of such slip pulses and, most importantly, their dynamic stability and dimensionality dependence remain rather poorly understood.

In this Letter, we establish a surprising connection between the two apparently disconnected classes of problems described above; we show that interfaces described by generic friction laws feature unstable steady-state slip pulses in the quasi-1D approximation. These unstable slip pulses of linear size $L^{*}$ and characteristic amplitude are hypothesized to serve as "critical nuclei" for the onset of rapid slip along quiescent interfaces, in a nonequilibrium analogy to equilibrium first-order phase transitions; that is, we propose an intimate relation between unstable slip pulses and earthquake nucleation.

These ideas are first quantitatively supported by dynamical quasi-1D calculations. Then the nucleation length $L^{*}$ is shown to exist also when 2D elastodynamics are considered, but the existence of a fracture mechanics [48,49] Griffith-like length $L_{G}$ gives rise to sustained slip pulses. The analysis culminates in a 2D phase diagram [21], highlighting the roles of unstable slip pulses in earthquake nucleation as a nonequilibrium first-order phase transition.

Spatially extended frictional systems and generic friction laws.-The dynamics of spatially extended frictional systems emerge from the coupling between the bulk dynamics 
of the deformable bodies in contact and the frictional interaction of the bodies along the contact interface. Bulk dynamics are described by continuum momentum balance $\rho \ddot{\boldsymbol{u}}(\boldsymbol{r}, t)=\nabla \cdot \boldsymbol{\sigma}(\boldsymbol{r}, t)$, where $\rho$ is the mass density, $\boldsymbol{u}$ and $\boldsymbol{r}$ are the d-dimensional displacement and position vector fields, respectively, $t$ is time, and $\sigma$ is the stress tensor field. $\boldsymbol{\sigma}$ is typically related to $\boldsymbol{u}$ through Hooke's law, resulting in bulk linear elastodynamics.

The interfacial constitutive (friction) law relates the slip velocity (the relative interfacial velocity), interfacial stresses, and the structural state of the interface. In $\mathrm{d}=2$, when sliding takes place along $x$ at $y=0$, the slip velocity reads $v(x, t) \equiv \dot{u}_{x}\left(x, y=0^{+}, t\right)-\dot{u}_{x}\left(x, y=0^{-}, t\right)$ ( + and - correspond to the upper and lower bodies, respectively), and the friction stress reads $\tau(x, t) \equiv$ $\sigma_{x y}(x, y=0, t)$. The structural state of the interface is described by an internal-state field $\phi(x, t)$, which satisfies its own evolution equation. Finally, $\tau(v, \phi)=$ $\sigma \operatorname{sgn}(v) f(|v|, \phi)$, where $\sigma \equiv-\sigma_{y y}(x, y=0, t)$ is the interfacial normal stress and $f(|v|, \phi)$ is the friction coefficient, and $\dot{\phi}=g(|v|, \phi)$. This is the rate-and-state friction constitutive framework [1,4,50,51].

We use constitutive functions $f(|v|, \phi)$ and $g(|v|, \phi)$ that capture the generic properties of frictional interfaces. First, we set $g(|v|, \phi)=1-|v| \phi / D[1,4,34,50-52]$, where $\phi$ represents the typical age or maturity of contact asperities that compose the spatially extended interface, such that $\phi=t$ accounts for frictional aging or healing in the absence of slip, $v=0$, and $\phi=D /|v|$ accounts for frictional rejuvenation over characteristic slip $D$ in the presence of slip, $v \neq 0$. Second, we use the function $f(|v|, \phi=D /|v|)$ [20,34] plotted in Fig. 1; this $N$-shaped steady-state friction curve

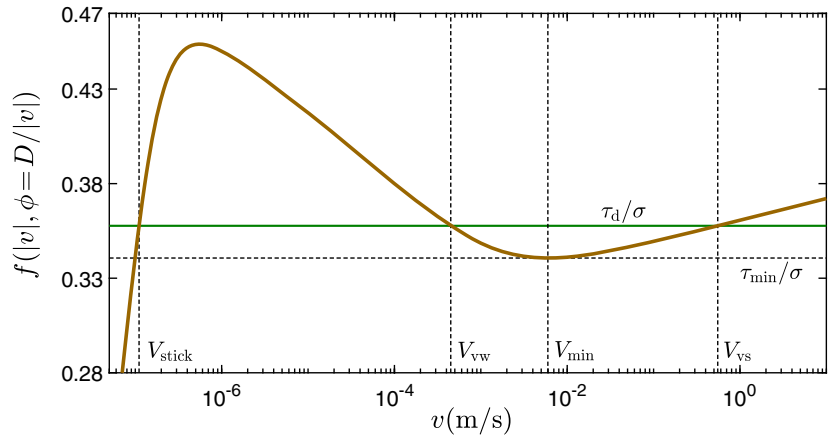

FIG. 1. A generic $N$-shaped steady-state friction coefficient $f=\tau / \sigma$ vs slip velocity $v$, featuring a minimum at $\left(V_{\min }, \tau_{\min } / \sigma\right)$. The solid horizontal line is the driving shear stress $\tau_{d}>\tau_{\min }$, intersecting the $N$-shaped friction law at three velocities: $V_{\text {stick }} \ll V_{\min }$ on the extremely low- $v$ velocitystrengthening branch, $V_{\mathrm{vw}}<V_{\min }$ on the velocity-weakening branch, and $V_{\mathrm{vs}}>V_{\min }$ on the high- $v$ velocity-strengthening branch. Note that the actual numbers used in the figure are characteristic of some laboratory experiments [34], but the results derived from them below are relevant to a broad range of materials and physical situations. features a velocity-strengthening branch at extremely small $v$ 's, essentially representing quiescent or locked interfacial states, a velocity-weakening branch at intermediate $v$ 's, and another velocity-strengthening branch beyond a high- $v$ minimum [34,53-55]. This generic friction curve is supported by extensive experiments and theoretical considerations [54].

The existence and properties of $1 D$ steady-state pulses.-The coupled interface-bulk problem defined above poses great mathematical challenges. To simplify things, we first consider two long and thin linear elastic bodies of height $H$ in frictional contact, such that $\rho \ddot{\boldsymbol{u}}=$ $\nabla \cdot \boldsymbol{\sigma}$ reduces to $[31,53]$

$$
H \bar{G}\left(c^{-2} \partial_{t t}-\partial_{x x}\right) u(x, t)=\tau_{d}-\tau[v(x, t), \phi(x, t)],
$$

where $u \equiv u_{x}, \bar{G}$ and $c$ are the effective shear modulus and wave speed [31,53], respectively, and $\tau_{d}$ is a constant driving stress (see Fig. 1). Quasi-1D traveling steady-state solutions then satisfy [34]

$$
\begin{gathered}
\bar{G} H c^{-1}\left(1-\beta^{2}\right) \beta^{-1} d v(\xi) / d \xi=\tau_{d}-\tau[v(\xi), \phi(\xi)], \\
\beta c d \phi(\xi) / d \xi=\phi(\xi) v(\xi) / D-1,
\end{gathered}
$$

where we defined a comoving coordinate $\xi \equiv x-\beta c t$, integrated out $u$, and eliminated partial time derivatives.

Steady-state pulses, featuring a steadily traveling slipping region (cf. Fig. 2), can be thought of as composed of interacting rupture and healing fronts that propagate at the same velocity. Such fronts connect velocity-strengthening (i.e., stable) solutions of $\tau(|v|, \phi=D /|v|)=\tau_{d}$; see Fig. 1 . In particular, in steady-state rupture fronts, the homogeneous $V_{\text {vs }}$ state invades the homogeneous $V_{\text {stick }}$ state $[27,30,32,33,53,56]$, both defined in Fig. 1, and vice versa for steady-state healing fronts. We found these solutions

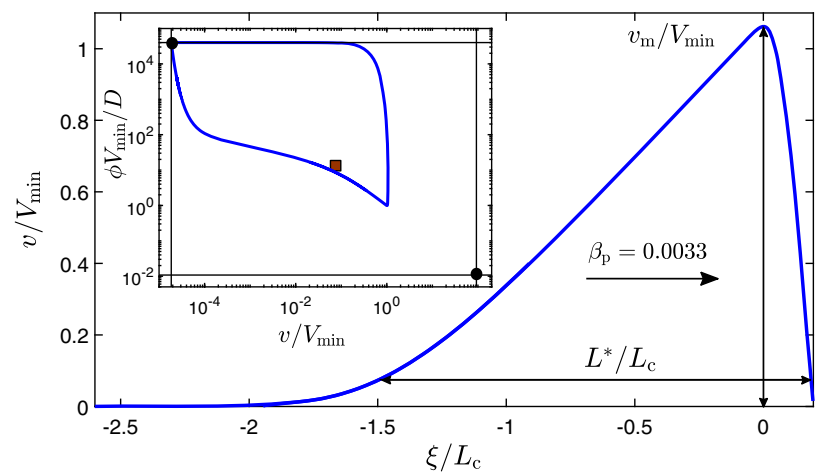

FIG. 2. A slip pulse solution corresponding to $\tau_{d}$ in Fig. 1, featuring a typical width $L^{*}$ (see the text for an exact definition) and a maximal slip velocity $v_{m}$ [34]. Length is measured in units of the velocity-weakening nucleation length $L_{c}[20,31,34]$ and velocity in units of $V_{\min }$. (Inset) The solution in the $\phi-v$ plane (the black circles correspond to the $V_{\text {stick }}$ and $V_{\text {vs }}$ fixed points and the brown square to the $V_{\mathrm{vw}}$ fixed point; see Fig. 1). 

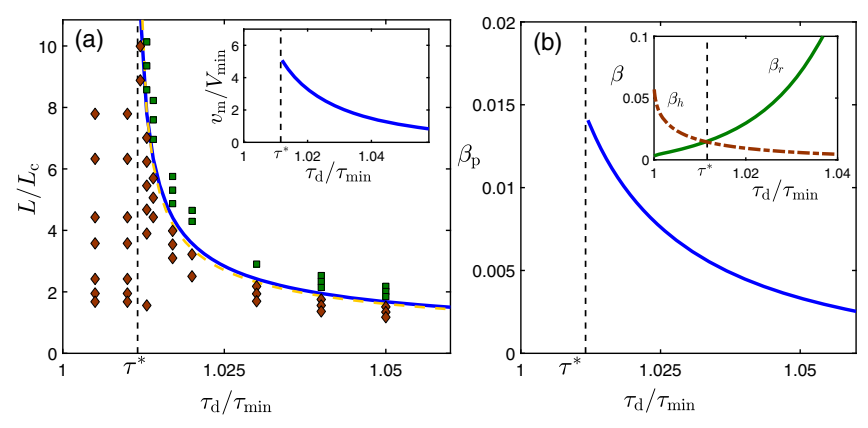

FIG. 3. (a) The normalized perturbation width $L / L_{c}$ vs $\tau_{d} / \tau_{\min }$. The theoretical prediction $L^{*}$ (solid blue line) separates dynamic perturbations that lead to nucleation (green squares) from those that decay (brown diamonds) (see the text for details) and closely follows the theoretical prediction $\sim 1 / \sqrt{\tau_{d}-\tau^{*}}$ (dashed yellow line) [34], where $\tau^{*}$ is marked by the vertical dashed line. (Inset) $v_{m} / V_{\min }$ vs $\tau_{d} / \tau_{\min }$. (b) The dimensionless pulse propagation velocity $\beta_{p}$ vs $\tau_{d} / \tau_{\min }$. (Inset) The dimensionless front propagation velocity $\beta$ (solid green line for rupture fronts $\beta_{r}$ and dashed-dotted brown line for healing fronts $\beta_{h}$ ) vs $\tau_{d} / \tau_{\min }$. The two curves intersect at $\tau^{*}$.

and calculated their dimensionless propagation velocity $\beta_{r, h}\left(\tau_{d}\right)$ (for rupture and healing fronts, respectively), as shown in the inset in Fig. 3(b). The two functions exhibit opposite trends and intersect at $\tau^{*}$ [33].

At $\tau_{d}=\tau^{*}$, rupture and healing fronts propagate at the same velocity $\left[\beta_{r}\left(\tau^{*}\right)=\beta_{h}\left(\tau^{*}\right)\right]$ and hence can be superimposed without interaction to form a pulse of infinite width. As $\tau_{d}$ is increased above $\tau^{*}$, the two fronts interact, leading to pulses of finite width $L^{*}\left(\tau_{d}\right)$ and propagation velocity $\beta_{p}\left(\tau_{d}\right)$. The existence of such pulses is explicitly demonstrated in Fig. 2. In Fig. 3(a), we show the pulse width $L^{*}\left(\tau_{d}\right)$, defined as the distance between the points at which the slip velocity drops to $V_{\mathrm{vw}}$ (cf. Fig. 1). A scaling theory predicts that $L^{*} / L_{c}$ diverges as $\sqrt{\tau^{*}} / \sqrt{\tau_{d}-\tau^{*}}$ for $\tau_{d} \rightarrow \tau^{*}$ [34], which is shown to quantitatively agree with the numerical results [dashed yellow line in Fig. 3(a)]. The maximal slip velocity $v_{m}\left(\tau_{d}\right)$ (see the definition in Fig. 2), plotted in the inset in Fig. 3(a), and the propagation velocity of slip pulses $\beta_{p}\left(\tau_{d}\right)$, plotted in Fig. 3(b), also increase with decreasing $\tau_{d}$.

Finally, we note that, while it is physically intuitive and appealing to think of slip pulses as interacting rupture and healing fronts, the existence of the latter is not a necessary condition for the existence of the former. That is, slip pulses exist also for steady-state friction laws that do not feature a minimum, for which steady-state fronts - and consequently a finite $\tau^{*}$ at which $L^{*}$ diverges—do not exist [34].

Unstable pulses as critical nuclei in a nonequilibrium first-order phase transition.-We next perform a numerical stability analysis of slip pulses. That is, we use steady-state pulse solutions as initial conditions and perturb them by slightly stretching or compressing the comoving coordinate $\xi$; see Fig. 4(a). Here, steady-state conditions are not enforced; i.e., we transform the equations to the comoving frame of reference without eliminating partial time derivatives [34] and track the time evolution of perturbations; see Figs. 4(b) and 4(c). It is observed that the perturbation with $L>L^{*}$ grows in amplitude and expands in size, while the perturbation with $L<L^{*}$ decays. That is, 1D steady-state pulses are intrinsically unstable.

The unstable nature of 1D steady-state slip pulses may imply that they play no role in fault dynamics. We propose, instead, that in fact they may serve as "critical nuclei" for the transition from an almost nonslipping state $V_{\text {stick }}$ to a slipping state $V_{\mathrm{vs}}$ in a nonequilibrium analogy to equilibrium first-order phase transitions [57]. That is, we propose that unstable slip pulses provide a dynamical mechanism for the nucleation of rapid slip along frictional interfaces
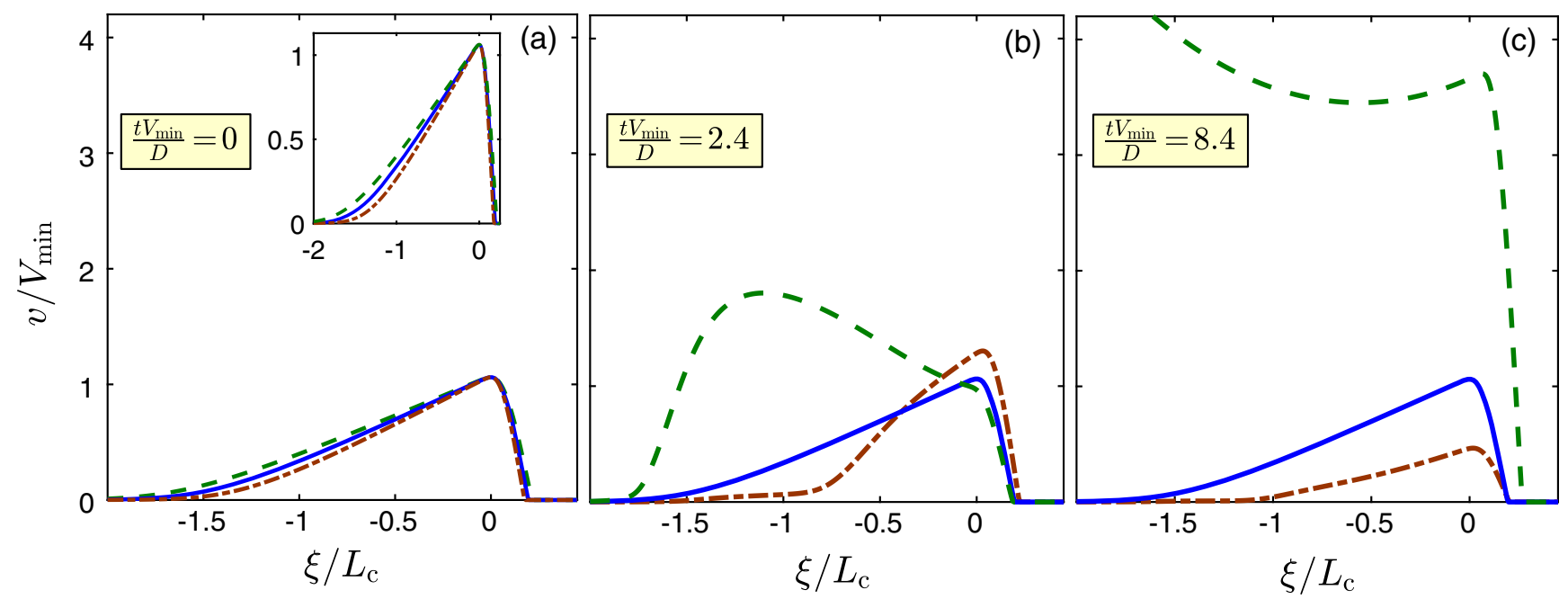

FIG. 4. (a) A steady-state pulse (solid blue line), which is slightly stretched (dashed green line), $L>L^{*}$, and compressed (dasheddotted brown line), $L<L^{*}$, at $t=0$. (Inset) Enlargement. As time progresses [(b),(c)], the stretched perturbation grows and expands, while the compressed one decays. 
that are initially at (or nearly at) rest, a regime that is not commonly studied in the literature. To test this idea, we introduced perturbations as initial conditions in the dynamical equations obtained by stretching $\left[L>L^{*}\left(\tau_{d}\right)\right]$ or compressing $\left[L<L^{*}\left(\tau_{d}\right)\right]$ the steady-state pulse solutions corresponding to $L^{*}\left(\tau_{d}\right)$ for each $\tau_{d}>\tau^{*}$ and by solving for a reduced $\tau_{d}$ using steady-state pulse solutions corresponding to $L=L^{*}$ for each $\tau_{d}<\tau^{*}$. We then tracked the system's evolution to determine whether the perturbations decay back to $V_{\text {stick }}$ or bring the system to $V_{\text {vs }}$.

The results, over a range of $L$ and $\tau_{d}$ values, are superimposed in Fig. 3(a). The figure provides compelling evidence that the theoretical prediction $L^{*}\left(\tau_{d}\right)$ indeed quantitatively predicts the fate of dynamic perturbations; i.e., perturbations with $L<L^{*}$ or $\tau_{d}<\tau^{*}$ (brown diamonds) decay back to $V_{\text {stick }}$, and those with $L>L^{*}$ (green squares) grow and bring the system to $V_{\mathrm{vs}}$, lending strong support to the proposed connection between unstable slip pulses and earthquake nucleation.

$2 D$ phase diagram: The Griffith-like length and sustained pulses.-The concepts and physical picture developed above are expected to be đ independent, and hence we expect the nucleation length $L^{*}\left(\tau_{d}\right)$ to exist also in đ $>1$. For đ $>1$, fronts and pulses are accompanied by a cracklike singularity near their edges [2,7,27,32,34], associated with a finite energy flux that is required to balance near-edge frictional dissipation per unit area, $G_{c}$ (an effective fracture energy) [32,34,58-61]. Consequently, for a given $G_{c}$, there exists a Griffith-like length $L_{G}\left(\tau_{d}\right)=$ $4 \mu \pi^{-1} G_{c}\left(\tau_{d}-\tau_{\text {res }}\right)^{-2}[48,49]$, where $\mu$ is the shear modulus and $\tau_{\text {res }}$ is the residual shear stress left behind the edge, below which no front or pulse propagation is possible.

Using $L^{*}\left(\tau_{d}\right)$ and $L_{G}\left(\tau_{d}\right)$, we can predict the salient features and topology of the $L-\tau_{d}$ phase diagram for đ $>1$. First, $L^{*} / L_{c}$ is predicted to diverge at a finite $\tau^{*}$ as $\tau^{*} /\left(\tau_{d}-\tau^{*}\right)$ for đ $>1$ [34]. As $L_{G}\left(\tau_{d}\right) \sim\left(\tau_{d}-\tau_{\text {res }}\right)^{-2}$ is a minimal condition for front and pulse propagation, we expect $L_{G}<L^{*}$ and $\tau_{\text {res }}<\tau^{*}$. Consequently, for $L<L_{G}$, we expect perturbations to decay without propagation, and hence no nucleation to occur, simply because no front or pulse can propagate. For $L>L^{*}$, we expect perturbations (of sufficiently large amplitude) to lead to the nucleation of the $V_{\mathrm{vs}}$ phase through propagating rupture fronts. For $L>$ $L_{G}$ and $\tau_{d}<\tau^{*}$, we expect no nucleation to occur but the decay of perturbations to be different from that in the regime $L<L_{G}$ and involve front or pulse propagation. Finally, for perturbations with $L_{G}<L<L^{*}$ under $\tau_{d}>\tau^{*}$, new dynamical modes that have no $1 \mathrm{D}$ analog might emerge.

To test these predictions, we performed spectral boundary integral method [62-64] calculations for infinite $₫=2$ systems under antiplane shear (mode III) [48]. The basic field in this problem, $u_{z}(x, y, t)(z \perp x, y)$, satisfies the bulk elastodynamic equation $\mu \nabla^{2} u_{z}=\rho \ddot{u}_{z}$, together with $v(x, t) \equiv \dot{u}_{z}\left(x, y=0^{+}, t\right)-\dot{u}_{z}\left(x, y=0^{-}, t\right)$ and $\tau(x, t) \equiv$ $\sigma_{y z}(x, y=0, t)=\mu \partial_{y} u_{z}(x, y=0, t)$. Furthermore, to test

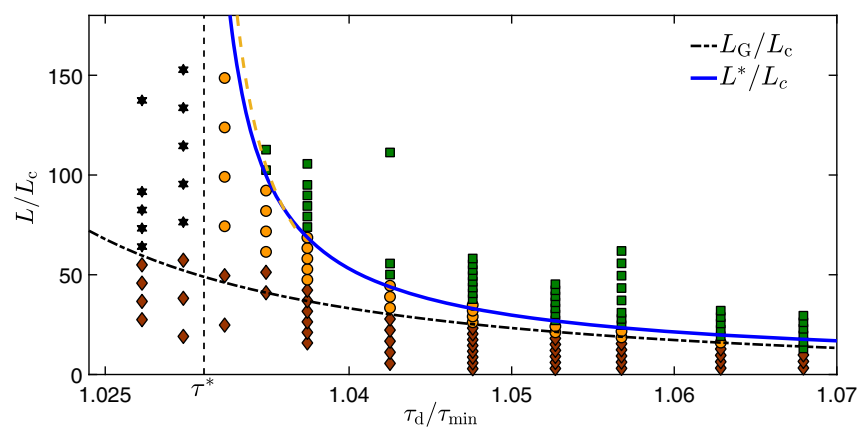

FIG. 5. A 2D phase diagram, the counterpart of the 1D phase diagram in Fig. 3(a). Dynamic perturbations that decay without propagation (brown diamonds) appear below the Griffith-like length $L_{G}\left(\tau_{d}\right)$ (dashed-dotted black line, obtained analytically; see the text for details). Dynamic perturbations that lead to nucleation (green squares) appear above $L^{*}\left(\tau_{d}\right)$ (solid blue line, estimated numerically), which follows the theoretical prediction $\sim 1 /\left(\tau_{d}-\tau^{*}\right)$ (dashed yellow line) when $\tau_{d}$ is close to $\tau^{*}$ (marked by the vertical dashed line) [34]. For $L>L_{G}$ and $\tau_{d}<\tau^{*}$, dynamical perturbations decay with some propagation of transient pulses (black hexagrams), and for $L_{G}<L<L^{*}$ and $\tau_{d}>\tau^{*}$, sustained pulses exist (orange circles; see the text for details). Movies are available in Ref. [34].

the robustness of the emerging physical picture for different types of initial perturbations, we consider here Gaussian perturbations (the perturbation's width $L$ is defined as 10 Gaussian standard deviations [34]), which are somewhat more generic. The results are presented in Fig. 5, where the theoretical prediction for $L_{G}\left(\tau_{d}\right)$ is added (dashed-dotted black line; details about the estimation of $G_{c}$ and $\tau_{\text {res }}$ can be found in Ref. [34]). First, we observe that $L_{G}\left(\tau_{d}\right)$ quantitatively predicts the boundary below which perturbations decay without propagation. Second, we observe that there exists a vertical boundary (dashed line), which is interpreted as $\tau^{*}$, such that no nucleation occurs for $\tau_{d}<\tau^{*}$, yet the decay for $L>L_{G}$ involves propagation of transient pulses, as predicted theoretically. Third, there exists a phase boundary (solid blue line), which appears to diverge at $\tau^{*}$ and hence interpreted as $L^{*}\left(\tau_{d}\right)$, above which nucleation occurs through rupture front propagation. The numerical $L^{*} / L_{c}$ line is consistent with the theoretical prediction (cf. dashed yellow line in Fig. 5). Movies are available in Ref. [34].

Finally, for $L_{G}<L<L^{*}$ and $\tau_{d}>\tau^{*}$, sustained pulses that do not appear to exist in 1D emerge [22,25]. In this dynamical regime, a pair of pulses moves away from one another, apparently indefinitely (see movie in Ref. [34]). While these pulses do not strictly reach steady-state conditions for computationally feasible system sizes [34], it is clear that they leave behind them a $V_{\text {stick }}$ state, and hence they do not lead to the nucleation of the $V_{\mathrm{vs}}$ phase. The results presented in Fig. 5 appear to be independent of the amplitude of perturbations, as long as it is larger than $V_{\mathrm{vw}}$ [34]. Note that, while in đ $=1$ no edge singularity exists, a Griffith-like length [which scales as $\left.\sim \sqrt{\bar{G} H G_{c}}\left(\tau_{d}-\tau_{\text {res }}\right)^{-1}\right]$ can still be formally defined using 
global energy balance considerations [34,65]. Yet, we found no trace for this length in our 1D phase diagram in Fig. 3(a).

The phase diagram in Fig. 5 may appear somewhat reminiscent of the computational results of Ref. [21], obtained in a large parametric study of in-plane (mode-II) dynamic rupture styles of faults featuring a finite shear strength and strong velocity-weakening friction. Yet, there are important differences between the two works. Most notably, we provide here a theoretical understanding of the phase boundaries $L^{*}\left(\tau_{d}\right)$ (associated with critical nuclei) and $L_{G}\left(\tau_{d}\right)$ (associated with a Griffith-like length), which is not developed in Ref. [21], and we directly relate $L^{*}\left(\tau_{d}\right)$ in $1 \mathrm{D}$ to steady-state slip pulses and their stability, which are not discussed in Ref. [21].

Concluding remarks and prospects.-We developed a comprehensive physical picture of rapid slip nucleation along quiescent frictional interfaces, highlighting the role of unstable slip pulses as critical nuclei of size $L^{*}$ in a nonequilibrium analogy to equilibrium first-order phase transitions. We also elucidated the conditions for the emergence of various propagative slippage modes (rupture styles), including rupture fronts, decaying pulses, transient pulses, and sustained pulses $[21,25]$. We stress that the physics behind the nucleation length $L^{*}$, associated with abrupt and stochastic processes, is qualitatively different from that of $L_{c}$, which is intrinsically related to a deterministic velocity-weakening linear frictional instability typically associated with precursory slow slip. Seismological evidence for such qualitatively different nucleation dynamics has been recently discussed [66] and should be further explored in the future.

E. B. and J.-F. M. acknowledge support from the Rothschild Caesarea Foundation. E. B. acknowledges support from the Israel Science Foundation (Grant No. 295/ 16). J.-F. M. and F. B. acknowledge support from the Swiss National Science Foundation (Grant No. 162569). M. A. thanks Y. Lubomirsky for assistance in numerical steadystate calculations in 1D. The authors thank E. Dunham and R. Viesca for useful discussions.

${ }^{*}$ E. A. B. and M. A. contributed equally.

[1] T. Baumberger and C. Caroli, Adv. Phys. 55, 279 (2006).

[2] Y. Ben-Zion, Rev. Geophys. 46, RG4006 (2008).

[3] A. Vanossi, N. Manini, M. Urbakh, S. Zapperi, and E. Tosatti, Rev. Mod. Phys. 85, 529 (2013).

[4] A. Ruina, J. Geophys. Res. Solid Earth 88, 10359 (1983).

[5] T. Yamashita and M. Ohnaka, J. Geophys. Res. Solid Earth 96, 8351 (1991).

[6] Y. Ben-Zion and J. R. Rice, J. Geophys. Res. Solid Earth 102, 17771 (1997).

[7] Y. Ben-Zion, J. Mech. Phys. Solids 49, 2209 (2001).

[8] J.-P. Ampuero, J.-P. Vilotte, and F. J. Sánchez-Sesma, J. Geophys. Res. Solid Earth 107, ESE 2-1 (2002).
[9] N. Lapusta and J. R. Rice, J. Geophys. Res. Solid Earth 108, 2205 (2003).

[10] K. Uenishi and J. R. Rice, J. Geophys. Res. Solid Earth 108, 2042 (2003).

[11] A. M. Rubin and J.-P. Ampuero, J. Geophys. Res. Solid Earth 110, B11312 (2005).

[12] J.-P. Ampuero and A. M. Rubin, J. Geophys. Res. Solid Earth 113, B01302 (2008).

[13] Y. Kaneko and N. Lapusta, J. Geophys. Res. Solid Earth 113, B12312 (2008).

[14] G. C. McLaskey and B. D. Kilgore, J. Geophys. Res. Solid Earth 118, 2982 (2013).

[15] S. Latour, A. Schubnel, S. Nielsen, R. Madariaga, and S. Vinciguerra, Geophys. Res. Lett. 40, 5064 (2013).

[16] R. C. Viesca, Phys. Rev. E 93, 060202 (2016).

[17] R. C. Viesca, Proc. R. Soc. A 472, 20160254 (2016).

[18] Y. Kaneko, S. B. Nielsen, and B. M. Carpenter, J. Geophys. Res. Solid Earth 121, 6071 (2016).

[19] Y. Kaneko, B. M. Carpenter, and S. B. Nielsen, Geophys. Res. Lett. 44, 162 (2017).

[20] M. Aldam, M. Weikamp, R. Spatschek, E. A. Brener, and E. Bouchbinder, Geophys. Res. Lett. 44, 11,390 (2017).

[21] A.-A. Gabriel, J.-P. Ampuero, L. A. Dalguer, and P. M. Mai, J. Geophys. Res. Solid Earth 117, B09311 (2012).

[22] G. Perrin, J. R. Rice, and G. Zheng, J. Mech. Phys. Solids 43, 1461 (1995).

[23] N. M. Beeler and T. E. Tullis, Bull. Seismol. Soc. Am. 86, 1130 (1996).

[24] A. Cochard and R. Madariaga, J. Geophys. Res. Solid Earth 101, 25321 (1996).

[25] G. Zheng and J. R. Rice, Bull. Seismol. Soc. Am. 88, 1466 (1998).

[26] S. B. Nielsen, J. M. Carlson, and K. B. Olsen, J. Geophys. Res. Solid Earth 105, 6069 (2000).

[27] A. Bizzarri and M. Cocco, J. Geophys. Res. 108, 2373 (2003).

[28] E. A. Brener, S. V. Malinin, and V. I. Marchenko, Eur. Phys. J. E 17, 101 (2005).

[29] A. M. Rubin and J.-P. Ampuero, J. Geophys. Res. 114, B11305 (2009).

[30] O. Ben-David, G. Cohen, and J. Fineberg, Science 330, 211 (2010).

[31] Y. Bar-Sinai, R. Spatschek, E. A. Brener, and E. Bouchbinder, Phys. Rev. E 88, 060403 (2013).

[32] I. Svetlizky and J. Fineberg, Nature (London) 509, 205 (2014).

[33] T. Putelat, J. H. Dawes, and A. R. Champneys, Proc. R. Soc. A 473, 20160606 (2017).

[34] See Supplemental Material at http://link.aps.org/ supplemental/10.1103/PhysRevLett.121.234302 for additional information, which includes Refs. [35-45].

[35] T. Putelat, J. H. Dawes, and J. R. Willis, J. Mech. Phys. Solids 59, 1062 (2011).

[36] C. Marone, Nature (London) 391, 69 (1998).

[37] Y. Estrin and Y. Bréchet, Pure Appl. Geophys. 147, 745 (1996).

[38] M. Nakatani and C. H. Scholz, J. Geophys. Res. Solid Earth 111, B12208 (2006).

[39] O. Ben-David, S. M. Rubinstein, and J. Fineberg, Nature (London) 463, 76 (2010). 
[40] M. Aldam, Y. Bar-Sinai, I. Svetlizky, E. A. Brener, J. Fineberg, and E. Bouchbinder, Phys. Rev. X 6, 041023 (2016).

[41] L. D. Landau and E. M. Lifshitz, Theory of Elasticity, 3rd ed. (Pergamon Press, London, 1986).

[42] J. Meiss, Differential Dynamical Systems (Society for Industrial and Applied Mathematics, Philadelphia, 2007).

[43] S. H. Strogatz, Nonlinear Dynamics and Chaos: With Applications to Physics, Biology, Chemistry, and Engineering, 2nd ed. (Westview Press, Boulder, CO, 2014).

[44] W. H. Press, S. A. Teukolsky, W. T. Vetterling, and B. P. Flannery, Numerical Recipes 3rd Edition: The Art of Scientic Computing (Cambridge University Press, Cambridge, England, 2007).

[45] W. E. Schiesser, The Numerical Method of Lines: Integration of Partial Differential Equations, 1st ed. (Academic Press, New York, 1991).

[46] J. L. Hardebeck and J. P. Loveless, Nat. Geosci. 11, 60 (2018).

[47] T. H. Heaton, Phys. Earth Planet. Inter. 64, 1 (1990).

[48] L. B. Freund, Dynamic Fracture Mechanics (Cambridge University Press, Cambridge, England, 1998).

[49] D. J. Andrews, J. Geophys. Res. 81, 3575 (1976).

[50] C. Marone, Annu. Rev. Earth Planet Sci. 26, 643 (1998).

[51] M. Nakatani, J. Geophys. Res. Solid Earth 106, 13347 (2001).

[52] P. Bhattacharya and A. M. Rubin, J. Geophys. Res. Solid Earth 119, 2272 (2014).
[53] Y. Bar Sinai, E. A. Brener, and E. Bouchbinder, Geophys. Res. Lett. 39, L03308 (2012).

[54] Y. Bar-Sinai, R. Spatschek, E. A. Brener, and E. Bouchbinder, J. Geophys. Res. Solid Earth 119, 1738 (2014).

[55] Y. Bar-Sinai, R. Spatschek, E. A. Brener, and E. Bouchbinder, Sci. Rep. 5, 7841 (2015).

[56] V. Rubino, A. J. Rosakis, and N. Lapusta, Nat. Commun. 8, 15991 (2017).

[57] H. E. Stanley, Phase Transitions and Critical Phenomena (Clarendon Press, Oxford, 1971).

[58] E. A. Brener and V. I. Marchenko, J. Exp. Theor. Phys. Lett. 76, 211 (2002).

[59] D. S. Kammer, M. Radiguet, J.-P. Ampuero, and J.-F. Molinari, Tribol. Lett. 57, 23 (2015).

[60] E. Bayart, I. Svetlizky, and J. Fineberg, Nat. Phys. 12, 166 (2015).

[61] I. Svetlizky, D. S. Kammer, E. Bayart, G. Cohen, and J. Fineberg, Phys. Rev. Lett. 118, 125501 (2017).

[62] P. H. Geubelle and J. R. Rice, J. Mech. Phys. Solids 43, 1791 (1995).

[63] J. W. Morrissey and P. H. Geubelle, Int. J. Numer. Methods Eng. 40, 1181 (1997).

[64] M. S. Breitenfeld and P. H. Geubelle, Int. J. Fract. 93, 13 (1998).

[65] A. Puzrin and L. Germanovich, Proc. R. Soc. A 461, 1199 (2005).

[66] J. Gomberg, Nat. Geosci. 11, 463 (2018). 